University of Wollongong

Research Online

Faculty of Engineering - Papers (Archive)

Faculty of Engineering and Information

Sciences

$1-1-2009$

\title{
Flux distribution at the cross section of stacked nanostructured magnetic ribbon
}

Zhi Wei Lin

University of Technology, Sydney

Jian Guo Zhu

University of Technology, Sydney

Youguang Guo

University of Technology

Tom H. Johansen

University of Wollongong, tomjo@uow.edu.au

Y. Yoshizawa

Follow this and additional works at: https://ro.uow.edu.au/engpapers

Part of the Engineering Commons

https://ro.uow.edu.au/engpapers/2797

\section{Recommended Citation}

Lin, Zhi Wei; Zhu, Jian Guo; Guo, Youguang; Johansen, Tom H.; and Yoshizawa, Y.: Flux distribution at the cross section of stacked nanostructured magnetic ribbon 2009, 3912-3914.

https://ro.uow.edu.au/engpapers/2797

Research Online is the open access institutional repository for the University of Wollongong. For further information contact the UOW Library: research-pubs@uow.edu.au 


\title{
Flux Distribution at the Cross Section of Stacked Nanostructured Magnetic Ribbon
}

\author{
Zhi Wei Lin ${ }^{1}$, Jian Guo Zhu ${ }^{1}$, Youguang Guo ${ }^{1}$, Tom H. Johansen ${ }^{2,3}$, and Y. Yoshizawa ${ }^{4}$ \\ ${ }^{1}$ Faculty of Engineering and Information Technology, University of Technology, Sydney, Broadway NSW 2007, Australia \\ ${ }^{2}$ Department of Physics, University of Oslo, Blindern Oslo, 0316, Norway \\ ${ }^{3}$ Institute for Superconducting and Electronic Materials (ISEM), University of Wollongong, NSW, Australia \\ ${ }^{1}$ Magnetic \& Electronic Materials Research Laboratory, Hitachi Metals, Ltd., Saitama 360-0843, Japan
}

\begin{abstract}
Conventional magnetization methods only measure the average magnetic properties over the whole sample. The information is insufficient to understand the local character. This paper presents the visualized magnetization process and its profiles in detail at the cross section of the FINEMET ribbon using advanced magnetooptical imaging technique. External magnetic fields were applied parallel to the surface of the ribbon. A magnetization curve was plotted by interpreting the profile of intensity of magnetooptical images. The profile indicates that the flux densities are higher at the cross section of the ribbons compared with those at the gaps between the ribbons. The flux lines mainly transmit inside the FINEMET ribbons though there are some stray fields between the ribbons.
\end{abstract}

Index Terms-Magnetic field measurement, soft magnetic materials.

\section{INTRODUCTION}

$\mathbf{N}$ ANOCRYSTALLINE magnetic materials $\mathrm{Fe}_{74.5-\mathrm{x}} \mathrm{Cu}_{\mathrm{x}}$ $\mathrm{Nb}_{3} \mathrm{Si}_{13.5} \mathrm{~B}_{9}$ [1], FINEMET registered trademark of $\mathrm{Hi}-$ tachi Metals, Ltd, are promising soft magnetic materials which normally are produced by annealing a melt-spun amorphous ribbon at about $550{ }^{\circ} \mathrm{C}$ for $1 \mathrm{~h}$. The interesting point is that the nanocrystals b.c.c. Fe-Si of 10-15 nm, which arise in heating treatment, disperse in the surrounding amorphous ferromagnetic matrix [2]-[4], and the nanocrystals are coupled through the amorphous matrix. Such exchange coupling results in their superior soft magnetic properties compared with the conventional soft magnetic materials. Therefore, nanocrystalline magnetic materials have attracted extensive attentions for wide range of technological applications [5]-[8] and replacement of conventional magnetic materials in transformers, motors, sensors, power electronics, electrical energy control/management systems, telecommunication equipment and pulse power devices [9].

In order to apply optimally FINEMET to advanced electromagnetic devices, the properties of the materials must be understood clearly. Normally the materials are characterized using conventional magnetization method. Such method can only measure the average properties over the whole volume of a sample [10], [11] and cannot provide local magnetization process. This paper presents the visualized local magnetization process by means of magnetooptical imaging (MOI) technique. The major advantage of the MOI technique is that the local details of magnetic structures ranging from micrometer to centimeter can be directly visualized. This study demonstrates that the MOI is a powerful technique to visualize the magnetization process of a FINEMET ribbon in detail.

Manuscript received March 04, 2009; revised April 19, 2009. Current version published September 18, 2009. Corresponding author: Z. W. Lin (e-mail: jacklin@eng.uts.edu.au).

Color versions of one or more of the figures in this paper are available online at http://ieeexplore.ieee.org.

Digital Object Identifier 10.1109/TMAG.2009.2023874



Fig. 1. The schematic of magnetooptical imaging system.

\section{EXPERIMENT SETUP}

Amorphous ribbon of the composite $\mathrm{Fe}_{74.5-\mathrm{x}} \mathrm{Cu}_{\mathrm{x}}$ $\mathrm{Nb}_{3} \mathrm{Si}_{13.5} \mathrm{~B}_{9}$ was prepared by a rapid quenching technique, following by annealing of the as-quenched ribbon at $550{ }^{\circ} \mathrm{C}$ without magnetic field for one hour at Argon atmosphere for crystallization. The size of the ribbon is $5 \mathrm{~mm}$ in width and 18 $\mu \mathrm{m}$ in thickness. To visualize the magnetization process at the cross section of the ribbon, the annealed ribbons were stacked and glued together to form a $5 \mathrm{~mm} \times 10 \mathrm{~mm} \times 10 \mathrm{~mm}$ cubic block. The cross section was polished carefully in order to obtain high resolution and quality magnetooptical image.

A magnetooptical imaging system, as shown in Fig. 1, was built based on the Faraday effect [12], that is, the rotation of the plane of polarization of a beam of linearly polarized light when the light passes through a magnetooptical media in the direction of magnetic field line. The review of MOI system is given by the [13]. The reflective mode was employed in this study since the sample is opaque. As a result of Faraday rotation, a grey scale image can be observed under a microscope with an analyzer orthogonal to the incident polarizer. The higher the local flux density is, the brighter the area (pixel) becomes. 


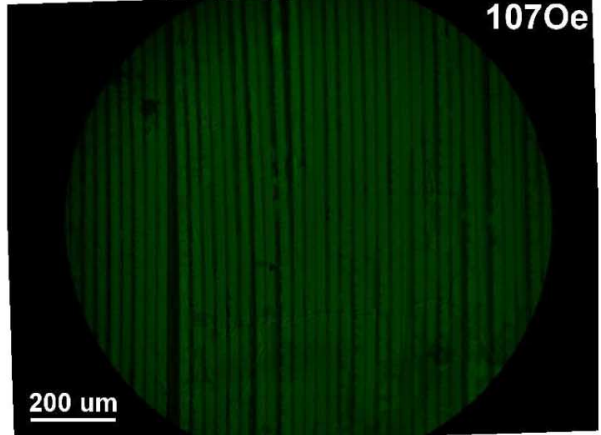

a



C

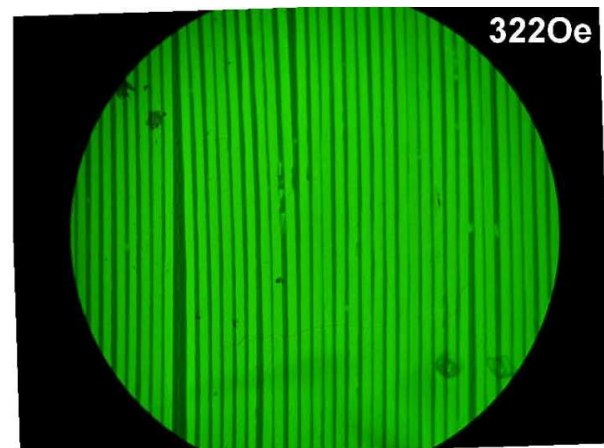

b

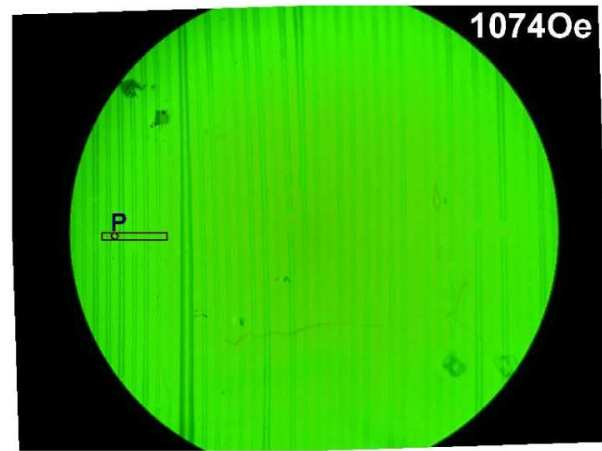

d

Fig. 2. Magnetooptical images of nanostructured magnetic ribbon at different external magnetic fields.

In this study, a green filter was employed to improve the contrast of the image since the rotation angle depends on the light frequency and reaches maximum at green light. The magnetooptical indicator film used in this study was an in-plane Bi substituted Yttrium Iron Garnet film $\left[\mathrm{Y}_{3} \mathrm{Fe}_{2}\left(\mathrm{FeO}_{4}\right)_{3}\right]$ deposited on a Gadolinium Gallium Garnet substrate. The indicator film was placed directly on the surface of the cross section with a slight mechanical pressure in order to improve spatial resolution. The image was captured by a CCD camera installed on the top of polarization microscope and transferred to a PC. The captured image size is 2272 by 1704 pixel, but the viewable field is about 1863 by 1704 pixel when the magnification of objective lens is 10. The magnetic field used to magnetize the sample was generated by a solenoid.

\section{RESULTS AND DISCUSSION}

In order to remove the image noise, ten magnetooptical images were taken at each applied field and averaged. Fig. 2 shows the averaged magnetooptical images when the demagnetized cubic sample was subjected to the increasing magnetic fields. The images show bright and dark stripes. The bright stripes correspond to the cross sections of the ribbons while the dark stripes indicate the gaps between ribbons. The width of the bright stripes is about $20 \mu \mathrm{m}$. The averaged intensity within each stripe is constant. In addition, the intensities of stripes within the image are similar, which means that the magnetization inside individual ribbon was homogeneous and all ribbons were magnetized similarly. The extent of magnetization increases with increasing applied field. Fig. 3 shows the averaged intensity profiles within the rectangle shown in Fig. 2(d). It is

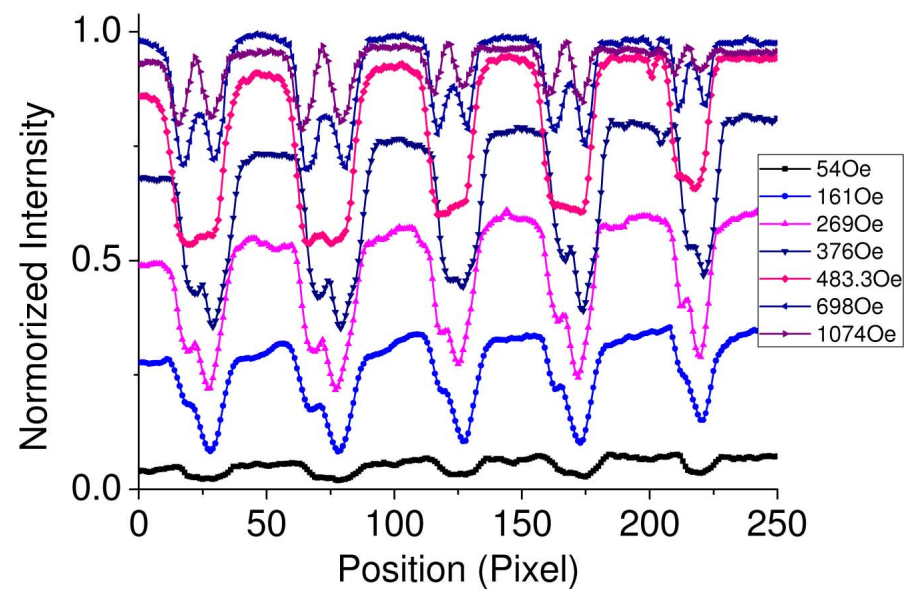

Fig. 3. Flux profiles along the bar shown in Fig. 2(d) at different external magnetic fields.

also noted that at higher fields, narrow and bright stripes appear between the ribbons. Such phenomenon is also indicated in the intensity profiles with a narrow peak between the wide peaks at the fields over $698 \mathrm{Oe}$. It is believed that these narrow peaks result from the superposition of neighboring stray field.

Due to the contribution of magnetization of the ribbon, the flux density at the cross section of each ribbon is higher than that at the gap, hence, more lights can pass through the analyzer. As a result, the stripe pattern is presented. With the increase of the applied fields, each ribbon is magnetized further, and hence the intensities over the ribbon areas increase. Fig. 4 plots the intensity profile as a function of the applied fields at the point of $P$ shown in Fig. 2(d) and inset is corresponding magnetization 




Fig. 4. Normalized light Intensity at point $P$ shown in Fig. 2(d) and inset is the magnetization curve derived from the intensity.

curve. It is found that the intensity increases quickly in the low applied fields range. However, it starts to decrease when the field is over the value of 644 Oe. Due to the limitation of the maximum magnetic field generated by the electromagnet and open magnetic loop, the sample is far from being saturated.

This phenomenon can be clearly understood from the law of Malus. Recall that the intensity of the light beam is proportional to the square of the amplitude. Since the polarizer and analyzer are orthogonal, the intensity of the reflective light $I$ passing the analyzer varies reaching the CCD chip of camera will be

$$
I=I_{m} \sin ^{2}(2 V B d)
$$

in which $I_{m}$ is the maximum value of the transmitted intensity, $V$ the Verdet constant of the indicator film, $d$ the thickness of the film, and $B$ the normal component of the saturated flux density of in-plane indicator film. The CCD camera then converts the light intensity into gray level value.

Following the applied field increasing, the flux density at the ribbon surface increases. It leads to the magnetization of the film rotating out of the film plane, that is, the $B$ increases, hence the light intensity over the ribbon increases. At a certain field, $H_{m}=644 \mathrm{Oe}$, the rotation angle approaches 90 degrees, that is, the indicator film is saturated, correspondingly, the intensity reaches the maximum. Over this field, the sample is magnetized further, but the intensity decreases gradually. Such decrease is caused by strong stray fields parallel to the indicator film at high fields [14]. In practice, the flux density is determined by comparing the measured intensity with the calibrated intensity curve.

\section{CONCLUSION}

This paper demonstrates that the magnetooptical imaging technique is a powerful method to visualize the magnetization process of a nanocrystalline magnetic ribbon. It is shown that the ribbons are magnetized uniformly. There are narrow flux density peaks between the ribbons, resulted from stray field of the ribbons. Based on the profiles of light density, a local magnetization curve can be plotted.

\section{REFERENCES}

[1] Y. Yoshizawa, S. Oguma, and K. Yamauchi, "New Fe-based soft magnetic alloys composed of ultrafine grain structure," J. Appl. Phys., vol. 64, pp. 6044-6046, 1988.

[2] G. Herzer, "Magnetization process in nanocrystalline ferromagnets," presented at the Materials Science \& Engineering A-Structural Materials Properties Microstructure \& Processing, Netherlands, Mar. 15, 1991.

[3] G. Herzer, "Grain-size dependence of coercivity and permeability in nanocrystalline ferromagnets," IEEE Trans. Magn., vol. 26, pp. 1397-1402, 1990.

[4] G. Herzer, "Soft magnetic nanocrystalline materials," Scripta Metall. Mater., vol. 33, pp. 1741-1756, 1995.

[5] C. Dudek, A. L. Adenot-Engelvin, F. Bertin, and O. Acher, "Engineering of the magnetic properties of FINEMET based nanocrystalline glass-coated microwires," J. Non-Crystal. Solids, vol. 353, pp. 925-927, Feb. 2007.

[6] P. Gupta, A. Gupta, V. Franco, and A. Conde, "Joule heating as a technique for obtaining uncoupled soft and hard magnetic phases in a finemet alloy," J. Appl. Phys., vol. 101, p. 033909-6, 2007.

[7] L. K. Varga, "Soft magnetic nanocomposites for high-frequency and high-temperature applications," J. Magn. Magn. Mater., vol. 316, pp. 442-447, Mar. 2007.

[8] T. Kulik, J. Ferenc, A. Kolano-Burian, X. B. Liang, and M. Kowalczyk, "Magnetically soft nanomaterials for high-temperature applications," J. Alloys Compounds, vol. 434, pp. 623-627, 2007.

[9] R. Hasegawa, "Present status of amorphous soft magnetic alloys," J. Magn. Magn. Mater. , vol. 215, pp. 240-245, 2000.

[10] J. G. Zhu, J. J. Zhong, V. S. Ramsden, and Y. G. Guo, "Power losses of soft magnetic composite materials under two- dimensional excitation," J. Appl. Phys, vol. 85, pp. 4403-4405, 1999.

[11] Z. W. Lin, J. G. Zhu, Y. G. Guo, X. L. Wang, and S. Y. Ding, "Threedimensional hysteresis of soft magnetic composite," J. Appl. Phys, vol. 99, pp. 08D909-, 2006.

[12] Z. W. Lin, J. W. Cochrane, G. J. Russell, S. X. Dou, and H. K. Liu, "Visualization of magnetic flux distribution in $\mathrm{Bi}(\mathrm{Pb})-2223 / \mathrm{Ag}$ multifilamentary tapes," Supercond. Sci. Technol., vol. 11, pp. 1017-1023, 1998.

[13] M. R. Koblischka and R. J. Wijngaarden, "Magneto-optical investigations of superconductors," Supercond. Sci. Technol., vol. 8, pp. 199-213, 1995.

[14] T. H. Johansen, M. Bazijevich, H. Bratsberg, Y. B. Galperin, P. E. Lindelof, Y. Shen, and P. Vase, "Direct observation of the current distribution in thin superconducting strips using magneto-optical imaging," Phys. Rev. B Condens. Matter, vol. 54, p. 16264, Dec. 1996. 